\title{
SURFACE MICROMACHINED ELECTROMAGNETICALLY RADIATING RF MEMS
}

\author{
Yong-Kyu Yoon, Bo Pan, Peter Kirby, John Papapolymerou, Manos Tentzeris, and Mark G. Allen \\ School of Electrical and Computer Engineering \\ Georgia Institute of Technology, Atlanta, GA 30332
}

\begin{abstract}
Increases in operational frequencies have reduced required RF radiator sizes, while simultaneously advances in 3-D fabrication technology have increased achievable thicknesses, such that surface micromachined radiators in the $30-300 \mathrm{GHz}$ range (millimeter wave range) are feasible in not only the horizontal dimension but also the vertical dimension. Much of the new fabrication technology enabling three-dimensional surface micromachining is based on photostructurable epoxies. Since at $\mathrm{GHz}$ frequencies, currents are confined to the outermost portions of conductors, small (micron-scale) thicknesses of metals deposited on three-dimensional epoxy scaffolds suffice to create RF subcomponents, the operation of which are indistinguishable from their solid metal counterparts. This concept has been demonstrated previously for simple passive elements such as inductors. In this work, the concept is extended to radiating structures. This approach offers the potential to create surface micromachined vertical radiating structures with higher potential performance than other MEMS-compatible solutions, e.g., conventional printed-circuit patch antennas. To illustrate this approach, a W-band $(75 \mathrm{GHz} 110 \mathrm{GHz})$, coplanar-waveguide (CPW)-fed, quarter-wavelength monopole antenna is designed, fabricated, and characterized. A monopole antenna with a height of $800 \mu \mathrm{m}$ is shown to radiate at $85 \mathrm{GHz}$ with $16 \mathrm{~dB}$ return loss (due to the radiation), which shows good agreement with simulation results.
\end{abstract}

\section{INTRODUCTION}

Millimeter-wave (MMW) devices are highly valued for their ability to provide very-broad-bandwidth wireless communication in both space and terrestrial applications. Examples include satellite, radar, mobile collision detection, imaging, and indoor local communications $[\mathbf{1}, \mathbf{2}]$. One of the key components for a wireless millimeter wave system is its radiating structure, i.e., its antenna. Currently, planar MMW antennas such as microstrip antennas or printed-circuit patch antennas are widely used due to their ease of manufacture, low cost, simple fabrication, and relative ease of integration with monolithic systems. However, patch antennas can suffer from relatively narrow bandwidth, substrate dielectric loss, mutual coupling with their substrate, and surface wave perturbation issues [3]. Although wire antennas (e.g., dipole or monopole antennas) or cavity antennas can be considered as alternatives to printed-circuit patch antennas because of their broad bandwidth, low loss, and reduced dependence on substrate, fabrication difficulty has prevented them from being efficiently implemented in a cost effective, integrated fashion.

Now we are at a unique intersection point in time in which increases in operation frequencies of RF systems have pushed characteristic sizes of RF subelements small enough, and advances in fabrication technologies have pushed achievable thicknesses of

Travel support has been generously provided by the Transducers Research Foundation and by the DARPA MEMS and DARPA BioFlips programs. surface micromachined components large enough, that an intersection has been achieved. Surface micromachined radiators in the 30-300 GHz range (millimeter wave range) are now feasible not only in the horizontal dimension but also in the vertical dimension, offering the potential to create radiating structures with better performance than, e.g., conventional printed-circuit patch antennas. Surface micromachining of these devices also offers the advantages of substrate independence as well as ease of integration with chips or substrates. The purpose of this work is to illustrate the capability of surface micromachining to create radiating structures for MMW applications.

An electromagnetic wave propagating through a good conductor attenuates very quickly in the depth direction of the conductor; the resultant electric current flows through the outermost portion of the conductor. Therefore, thin (micron-scale) metal layers deposited on three-dimensional (3-D) epoxy backbones should be sufficient to create RF components whose electrical characteristics are equivalent to those of their solid metal counterparts. This concept has been demonstrated previously for non-radiating passive elements: RF MEMS based on epoxy-core conductors [4], in which fabrication of a high-aspect-ratio RF inductor with height on the order of $1 \mathrm{~mm}$ has been successfully demonstrated. The three-dimensionally (3-D) complex structures achievable using mm-thickness, photostructurable resists [5-8] can now also create attractive geometries for high frequency radiators.

In this work, to illustrate this approach, a W-band $(75 \mathrm{GHz} \sim$ $110 \mathrm{GHz}$ ), coplanar-waveguide (CPW)-fed, quarter-wavelength monopole antenna is designed, fabricated, and characterized. This antenna has several unique properties. First, it is fed using a coplanar waveguide, which provides easy connectivity to other components and ease of fabrication compared to a via-throughsubstrate approach. Second, the epoxy core technique is used to provide a simple and efficient methodology for the transition from 2-D CPW structure to 3-D monopole. Third, since the fabricated monopole antenna is achieved by a low-temperature, foundrycompatible process, fully-integrated millimeter wave systems are feasible.

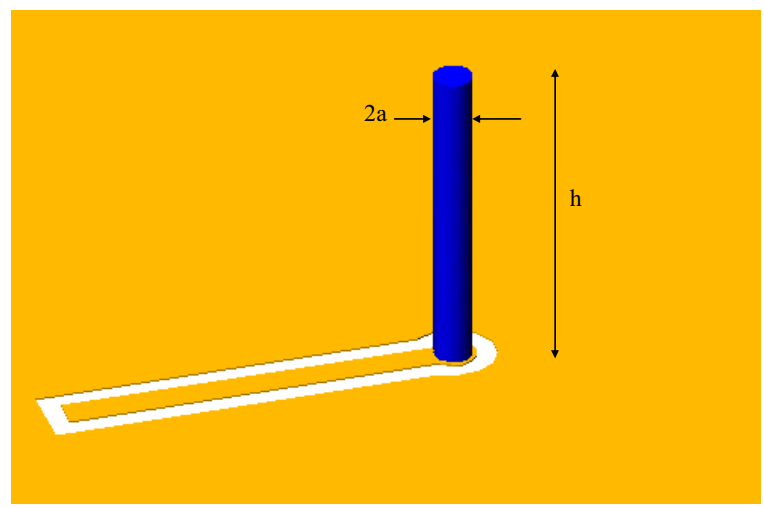

Figure 1. Schematic of a coplanar-waveguide fed quarterwavelength monopole antenna. 


\section{DESIGN AND SIMULATION}

To design an integrated type monopole antenna, fabrication limitations and their associated effect on antenna frequency must be taken into account. Limitations exist for both achievable aspect ratio (height to diameter ratio, $h / 2 a$ in Figure 1) as well as achievable absolute monopole height corresponding to the frequency range of interest.

The height of a quarter wave monopole in $\mathrm{W}$-band $(75 \mathrm{GHz} \sim$ $110 \mathrm{GHz}$ ) is in the range of $1 \mathrm{~mm}$ to $680 \mu \mathrm{m}$. The feasibility of tall $\mathrm{RF}$ conductor fabrication (up to $1 \mathrm{~mm}$ ) with aspect ratios of 10 to 15 was demonstrated in a previous work [4]. In practice, the monopole is cylindrical with a diameter of $2 a$ rather than an ideal wire with zero thickness. The nonideal cylindrical monopole therefore has an inductive reactance term attributable to the nonzero width of the conductor when it is driven at the radiating resonance frequency of an ideal monopole of the same height. This reactance term results in the nonideal monopole having its actual resonance at a slightly lower frequency than that of an ideal monopole. Alternatively, if a particular resonant frequency is desired, the monopole length can be reduced to achieve the ideal monopole resonant frequency. The magnitude of this height correction depends on the aspect ratio of the cylinder [9]; for example, in the case of a typical fabrication-limited aspect ratio of 10 , the height $h$ of the 'quarter-wave' monopole is given to be $0.228 \lambda$. The height of the quarter-wavelength monopole required for radiation resonance in W-band is plotted in Figure 2, where the dotted line and the solid line represent an uncompensated ideal monopole and a compensated practical monopole with an aspect ratio of 10 , respectively. In addition to the height corrections discussed above, it should be noted that the thicker the cylindrical monopole (i.e., the lower the aspect ratio), the wider its bandwidth becomes, and the less sharp its band-selectivity becomes.

Although the actual radiation resistance should be calculated using methods that take into account parasitics, driving elements, and imperfect ground planes, it is instructive to consider some simplified design equations for the micromachined monopole. For a quarter-wavelength monopole antenna of aspect ratio less than infinity over an ideal ground plane, the empirical radiation resistance $R_{A}$ is given by Equation 1 [10]:

$$
R_{A}=12.35(2 \pi h / \lambda)^{2.4}
$$

Using a fabrication-limited aspect ratio of 10, and a resultant height $h$ of $0.228 \lambda$, the predicted radiation resistance is $29.3 \Omega$. The ohmic resistance $R_{\text {ohmic }}$ of the antenna conductor can be calculated using Equation 2:

$$
R_{\text {ohmic }}[\Omega]=R_{s} \frac{h}{2 \pi a},
$$

where $R_{s}$ is the surface resistance or sheet resistance defined as:

$$
R_{s}=\sqrt{\frac{\omega \mu}{2 \sigma}},
$$

and $\omega, \mu$, and $\sigma$ are the radian frequency, permeability of the conductor, and conductivity of the conductor, respectively.

If the wire is made of gold $\left(\sigma=4.1 \times 10^{7} \mathrm{~S} / \mathrm{m}\right)$, the surface resistance $R_{s}$ at $85 \mathrm{GHz}$ is calculated to be $0.092 \Omega / \mathrm{sq}$. With $h$ of $800 \mu \mathrm{m}$ and $a$ of $40 \mu \mathrm{m}, R_{\text {ohmic }}$ is $0.29 \Omega$. The ohmic resistance of the wire is less than $1 \%$ of the radiation resistance. Therefore, the

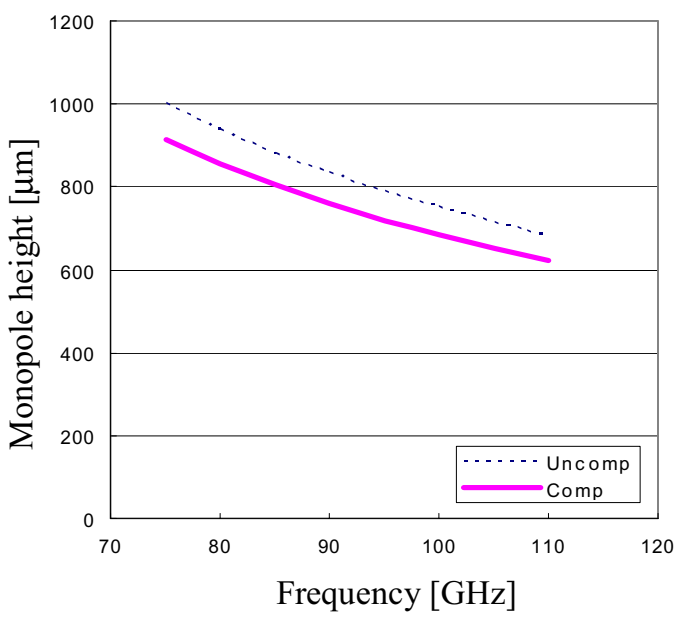

Figure 2. Height of a monopole required resonance radiating at W-band: Uncomp and Comp represent the height of the ideal monopole and that of a cylindrical monopole, respectively, where the aspect ratio of height to diameter is 10.

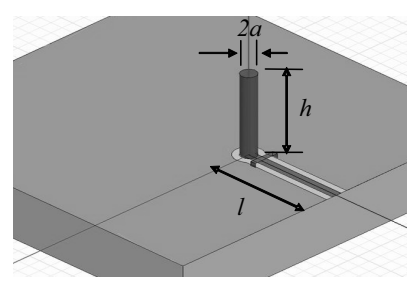

(a)

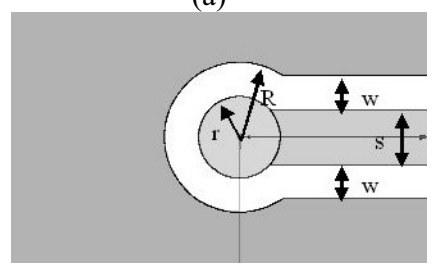

(b)

Figure 3. Monopole antenna fed through coplanar waveguide $(C P W)$ : (a) overall view; (b) top view showing geometrical parameters.

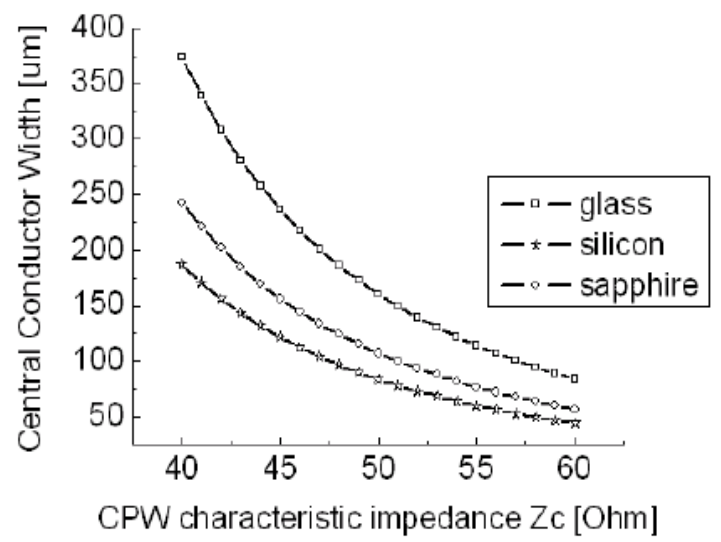

Figure 4. Central conductor width of $C P W$ according to characteristic impedance $Z c$ on different substrates (gap width is fixed to $50 \mu \mathrm{m}$ ). 
antenna input resistance can be approximated by the antenna radiation resistance in resonance mode.

Another consideration of the monopole design is the scheme required to feed it. A monopole is usually fed against a large solid ground plane, which requires via fabrication through the substrate. Instead, here a coplanar-waveguide (CPW) feeding scheme is used as shown in Figure 3, resulting in a via-free and a low-cost solution for an integrated RF transceiver system. The characteristic impedance of CPW lines on silicon $\left(\varepsilon_{r}=11.7\right)$, sapphire $\left(\varepsilon_{r}=10\right)$, and glass $\left(\varepsilon_{r}=7.8\right)$ has been calculated as a function of the normalized center conductor width, using the LineCal functionality of the Agilent advanced design system (ADS) and is shown in Figure 4. The gap width $w$ is fixed to $50 \mu \mathrm{m}$ and the ground is assumed to be infinite. The calculated characteristic impedance on these substrates is between $50 \Omega$ and $60 \Omega$ with a central conductor width $s$ of $80 \mu \mathrm{m}$, which is appropriate for the impedance matching to the system (its characteristic impedance is usually $50 \Omega$ ) but may not be good for antenna input impedance matching, which could be solved by CPW geometry optimization.

For a more synthetic analysis, the monopole antenna and the feeding CPW line can be analyzed as a whole using numerical software. Input return loss and radiation pattern have been simulated using the Ansoft high frequency system simulator (HFSS) 9.0 with the parameters listed below on various substrates.

The radius of ground aperture $R: 110 \mu \mathrm{m}$

The radius of bottom pad of monopole $r: 60 \mu \mathrm{m}$

Gap between the signal and the ground lines $w: 50 \mu \mathrm{m}$

Central conductor line width $s: 80 \mu \mathrm{m}$

Monopole width $2 a: 80 \mu \mathrm{m}$

Monopole height $h: 800 \mu \mathrm{m}$

CPW line length $l: 1 \mathrm{~mm}$

The simulation results for a glass substrate are plotted in

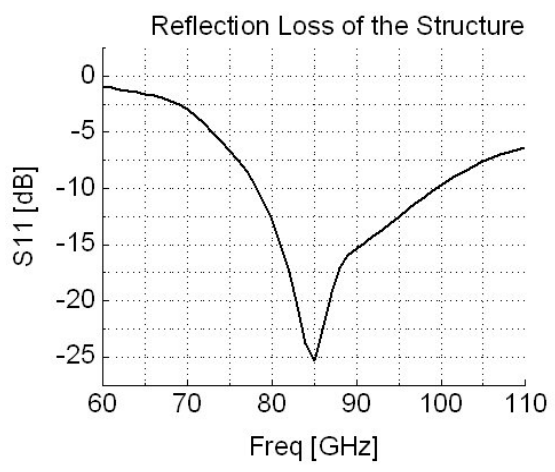

(a)

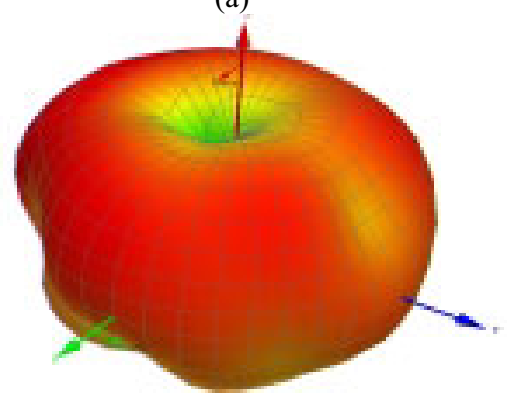

(b)

Figure 5. Simulated (a) input return loss and (b) radiation pattern for the monopole on soda lime glass using Ansoft HFSS.
Figure 5. The monopole shows resonance at a frequency of $85 \mathrm{GHz}$, and the far-field radiation pattern shows an omnidirectional and quite symmetric pattern, as expected.

\section{FABRICATION AND MEASUREMENT}

A polymer core conductor fabrication technique [4] has been adopted for the high-aspect-ratio monopole structure. A photodefinable epoxy, SU-8 (Microchem, Inc.), which is favorable for high-aspect-ratio micro patterning [11], is used for the monopole backbone, and electroplated gold is used for the electrical conductive path. The skin depth of gold in the W-band $(75 \mathrm{GHz} \sim 110 \mathrm{GHz})$ is in the range of $0.30 \mu \mathrm{m}$ to $0.24 \mu \mathrm{m}$. In general, 5 times the skin depth is considered to be sufficient to minimize the RF conductor loss and thereby not degrade the electrical performance. In this case a gold layer of approximately $1.5 \mu \mathrm{m}$ was used.

Figure 6 details the fabrication process. A chromium coated soda-lime glass (Telic Co.) is used as a substrate. Chromium is patterned for the monopole column definition using standard photolithography (a). SU-8 epoxy ( $800 \mu \mathrm{m}$ thick) is coated on the substrate to a thickness that will ultimately define the monopole height. The SU-8 is exposed to a UV source from the substrate side to obtain a uniform column latent pattern. Alternatively, a front side exposure can be used if the substrate is opaque ( $\mathrm{Si}$, GaAs, etc.) (b). The latent pattern is developed. Metal deposition of titanium and copper using a DC sputterer is carried out to form a conformal seed layer. Thin SU-8 $(5 \mu \mathrm{m})$ is spin-coated and patterned for the definition of the signal path as well as ground pads using proximity photolithography due to the uneven surface topography (c). Gold of $2 \mu \mathrm{m}$ thickness is uniformly electrodeposited through the bottom mold as well as over the column surface. The thin SU-8, the seed layer, and the bottom chromium layer are removed sequentially to complete the process (d). For fabrication simplicity, a two-mask process has been described above. In order to obtain more accurate bottom electrode

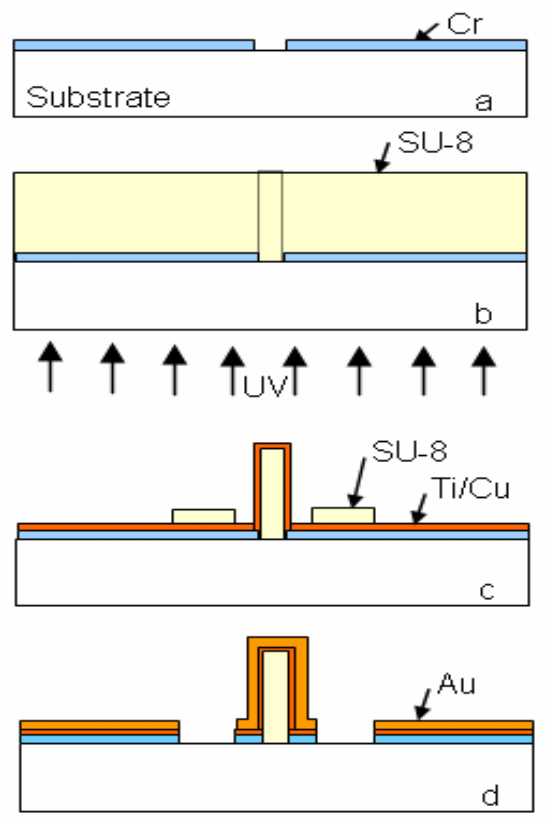

Figure 6. Fabrication process for a monopole antenna using epoxy core conductor scheme. 
dimensions for the signal and the ground lines, bottom line metallization can be performed separately from the monopole metallization with an additional mask step. Since the maximum temperature of any fabrication step is below $100{ }^{\circ} \mathrm{C}$, the process is CMOS compatible and easily integrable with a variety of substrates. Figure $7 \mathrm{a}$ and $7 \mathrm{~b}$ show a photomicrograph of a fabricated CPW-fed monopole antenna array $(3 \times 3)$ and an SEM image of a single monopole antenna, respectively. The fabricated structure is measured to be approximately $800 \mu \mathrm{m}$ tall.

An Agilent $8510 \mathrm{C}$ vector network analyzer connected to a Cascade GSG 150 probe station has been used for s-parameter measurement after a standard SOLT calibration between $50 \mathrm{GHz}$ and $110 \mathrm{GHz}$. Figure 8 shows measured and simulated return loss of a single monopole antenna from $50 \mathrm{GHz}$ to $110 \mathrm{GHz}$. The measurements have been performed with a monopole with a height of $800 \mu \mathrm{m}$. A return loss of $16 \mathrm{~dB}$ was measured for the monopole resonating at $85 \mathrm{GHz}$. Good agreement between simulation and measurement is observed for the $800 \mu \mathrm{m}$ tall sample. Radiation pattern measurements are currently underway.

\section{CONCLUSIONS}

A previously-developed epoxy core conductor fabrication concept is extended to millimeter wave applications with radiating structures. To demonstrate the process feasibility, a W-band micromachined monopole, which is vertically mounted on a glass substrate, is reported. A 2-D to 3-D feeding scheme is also proposed and optimized by simulation using a high frequency system simulator. Both the simulation and measurement results agree well. A return loss of $16 \mathrm{~dB}$ has been measured for a monopole of $800 \mu \mathrm{m}$ thick, resonating at $85 \mathrm{GHz}$. This micromachined monopole, as well as more complex geometries based on this fabrication technology, may have application in lowcost broadband compact millimeter wave communication systems.

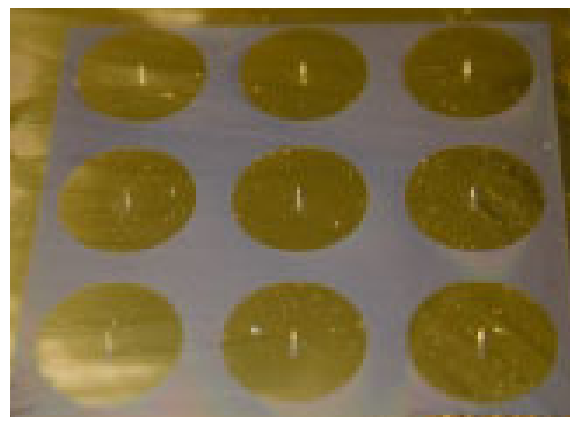

(a)

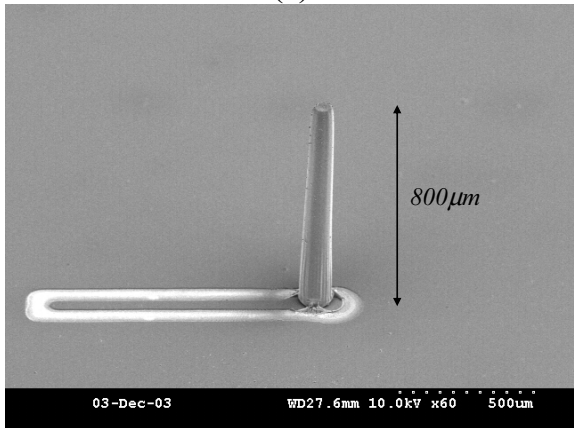

(b)

Figure 7. Fabricated CPW-fed monopole antenna: (a) photomicrograph of $3 X 3$ monopole array; (b) SEM image of $800 \mu \mathrm{m}$ tall monopole.

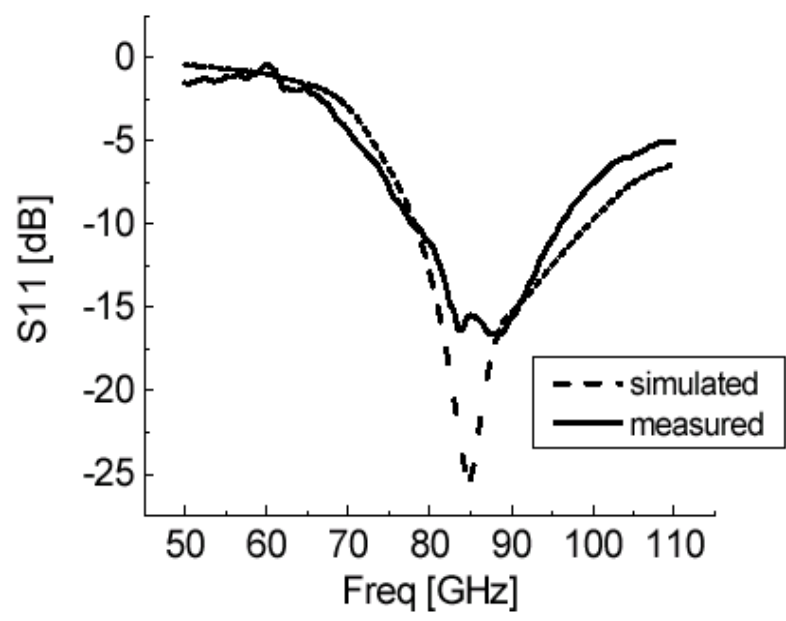

Figure 8. Measured and simulated reflection power (S11) for a monopole with a pole height of $800 \mu \mathrm{m}$.

\section{REFERENCES}

1. F.K. Schwering, "Millimeter Wave Antennss," Proceedings of the IEEE, Vol. 80, No. 1, pp. 92-102, January, 1992.

2. G.M. Rebeiz, "Millimeter-Wave and Terahertz Integrated Circuit Antennas," Proceedings of the IEEE, Vol. 80, No. 11, pp. 1748-1770, November, 1992.

3. D.M. Pozar, "Considerations for Millimeter Wave Printed Antennas," IEEE Trans. Antennas and Propagation, Vol. AP-31, No. 5, pp. 740-747, September, 1983.

4. Y.-K. Yoon, J.-W. Park, and M.G. Allen, "RF MEMS Based on Epoxy-Core Conductors," Digest of the 2002 Solid-State Sensor, Actuator, and Microsystems Workshop, Hilton Head Island, South Carolina, pp. 374-375, 2002

5. M. Han, W. Lee, S.-K. Lee, S.S. Lee, "Fabrication of 3D Microstructures with Inclined/Rotated UV Lithography," Proceedings of the IEEE Microelectromechanical Systems Conference (MEMS), Kyoto, Japan, pp. 554-557, 2003.

6. H. Sato, T. Kakinuma, J.S. Go, and S. Shoji, "A Novel Fabrication of In-channel 3-D Micromesh Structure Using Maskless Multi-Angle Exposure and Its Microfilter Application," Proceedings of the IEEE Microelectromechanical Systems Conference (MEMS), Kyoto, Japan, pp.223-226, 2003.

7. Yong-Kyu Yoon, Jung-Hwan Park, Florent Cros, and Mark G. Allen, "Integrated Vertical Screen Microfilter System Using Inclined SU-8 Structures," Proceedings of the IEEE Microelectromechanical Systems Conference (MEMS), Kyoto, Japan, pp.227-230, 2003.

8. Yoonsu Choi, R. Powers, Y.-K Yoon, M.G. Allen, "A ThreeDimensional Microfluidic Network for Cellular Perfusion," The $7^{\text {th }}$ International Conference on Miniaturized Chemical and BioChemical Analysis Systems (MicroTAS2003), pp. 1001-1004, Oct. 2003.

9. W.L. Stutzman and G.A. Thiele, Antenna Theory and Design, Chapters 2 and 5, John Wiley \& Sons, Inc., New York, 1998.

10. R.F. Schwartz, "Input Impedance of a Dipole or Monopole," Microwave Journal, vol. 15, p.22, Dec. 1972.

11. H. Lorenz, M. Despont, N. Fahrni, J. Brugger, P. Vettiger, P. Renaud, "High aspect ratio, ultrathick, negative-tone near-UV photoresist and its applications for MEMS," Sensors and Actuators, Part A, vol. 64, pp-33-39, 1998. 